\title{
Generation dispatch method based on maximum entropy principle for power systems with high penetration of wind power
}

\author{
Qiaoyan BIAN ${ }^{1}$, Yutao QIU ${ }^{2}$, Wenlian WU ${ }^{1}$, Huanhai XIN ${ }^{3}$, \\ Xuhua $\mathrm{FU}^{4}$
}

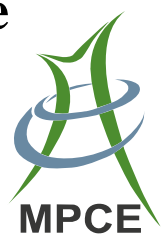

\begin{abstract}
This paper proposes a generation dispatch model based on the maximum entropy principle. The objective is to find an optimal generation dispatch strategy that minimizes the generation cost and satisfies the security constraints of power systems, while taking into account the uncertainty of wind power. Since in many situations, only partial information of the probabilistic variables can be obtained, the maximum entropy principle is introduced to find the most likely realized probability distributions of the power flow, thus providing an accurate probabilistic circumstance to solve the generation dispatch model. The proposed method is tested on the IEEE 39-bus system, and
\end{abstract}

CrossCheck date: 4 April 2018

Received: 28 December 2016/Accepted: 4 April 2018/Published online: 6 July 2018

(C) The Author(s) 2018

$\triangle$ Qiaoyan BIAN

bianqiaoyan@zju.edu.cn

Yutao QIU

bianqiaoyan@163.com

Wenlian WU

zjhzwwl@163.com

Huanhai XIN

xinhh@zju.edu.cn

Xuhua FU

littlebian@126.com

1 Hangzhou Power Supply Company of State Grid Zhejiang Electric Power Company, Hangzhou 310009, China

2 State Grid Zhejiang Electric Power Company, Hangzhou 310009, China

3 College of Electrical Engineering, Zhejiang University, Hangzhou 310027, China

4 Zhejiang Power Economic Research Institute of State Grid, Hangzhou 310027, China is compared with the methodologies based on Monte Carlo simulation and Gram-Charlier expansions.

Keywords Generation dispatch, Strategy, Maximum entropy, Probability distribution, Cumulant

\section{Introduction}

Nowadays, due to the problem of global warming and the transformation of energy systems, renewable energy including wind power is increasingly being integrated into the power systems. Wind power is clean and sustainable, however, the intermittency and variability of wind power generation poses big challenges to power system operations. In order to facilitate the integration of large-scale wind power, the fast-responding conventional generators need to compensate the power mismatch caused by the variability of wind power $[1,2]$.

The object of power system generation dispatch is to maintain the balance of power demand and supply at a minimum generation cost, considering operational constraints such as transmission limits and generation ramping rates. Without high penetration of intermittent renewable resources, power mismatch is mainly caused by the fluctuation of power demand, which has been studied for several decades. With the integration of large-scale wind power, both the demand side and the supply side exhibit uncertainties, which may have detrimental effects on the power balance. The fluctuations of wind power and load need to be smoothed by compensation from conventional generation. This can be achieved through generation dispatching operations, by means of coal-fired generator reserve scheduling which can use automatic generation 
control (AGC) [3], small-size hydropower unit control or fast-responding gas generator startup/shutdown [4, 5].

Deterministic power flow approaches used to be a common tool for power system operation scheduling. However, since the output of wind power is hard to predict accurately, and short-term wind power forecast results are always expressed in a statistical way [6], deterministic power flow approaches may not be adequate for generation dispatch with wind power [7]. In the presence of uncertain sustainable energy resources, probabilistic power flow (PPF) approaches are generally used for generation dispatch, such as the probabilistic security-constrained optimal power flow method in [7], which considers the impact of uncertainty sources, and the dispatch, network and security constraints in pre- and post-contingency states. The stochastic dynamic programming approach in [8] aims to find optimal solutions for unit commitment and dispatch, so as to minimize the operating costs with uncertain wind generation. The chance-constrained optimization approach is applied in [3] to find an optimal generation dispatch strategy considering variable photovoltaic resources.

PPF approaches usually employ both simulation techniques and analytical techniques to solve the optimization problem. Simulation techniques, such as Monte Carlo simulation in [7, 9], always need to deal with a large sample set and thus require a large computation effort. Analytical techniques include but are not limited to GramCharlier (GC) expansion in [3, 10, 11], Cornish-Fisher (CF) expansion in [12-14], the point estimate method in [15-17], as well as the direct or fast Fourier transformation (FFT) in [18, 19]. Both GC and CF expansions are combined with the cumulant method which provides cumulant information of power flow solutions, then GC expansion writes a probability density function (PDF) as a series composed of standard normal distribution derivatives and GC coefficients using the cumulants, and CF expansion expresses a cumulative density function (CDF) in terms of the quantile of a normal distribution and the cumulants. The point estimate method weights the load-flow solution estimates to calculate the moments of the solution distributions, and then approximates the probability distribution from the moments.

In PPF analysis, in many cases, only partial information about the random variables can be obtained [20]. For example, after the cumulant calculation procedures, only several orders of cumulants or moments can be collected for the power flow solutions; generally, even the input statistical description of wind power is not accurate and complete [6]. Therefore, it is difficult to calculate the exact probability distribution for the uncertain variables in generation dispatch [21]. Several PPF algorithms, such as the GC expansion and CF expansion, can be used to approximate the probability distribution of the uncertain variables, but the results are often based on arbitrary estimates of the unknown distribution.

In this paper, the maximum entropy (ME) principle is introduced to the generation dispatch problem. The basic idea is to find the most likely realized probability distribution, so as to provide a circumstance that is the closest to the actual probabilistic situation. Using this most likely distribution, the optimal dispatch strategy is solved to minimize the generation cost of compensating the fluctuation of wind power and system load.

The rest of this paper is organized as follow: Section 2 introduces the model of generation dispatch. PPF analysis based on the principle of maximum entropy is shown in Section 3. The optimal generation dispatch strategy is solved by a chance-constrained programming model in Sections 4 and 5 presents a case study based on the benchmark IEEE 39 bus system. Finally, Section 6 concludes this paper.

\section{Model for generation dispatch}

In this section, the impact of wind power variation on the power system reliability is described by the linearized power flow model. A generation dispatch model is presented to calculate the reaction of conventional generators to the variation of wind power and system load.

\subsection{Power flow model with wind power variation}

The generic power flow model for power injections and line flows is written as follows [22]:

$$
\left\{\begin{array}{l}
P_{i}=V_{i} \sum_{j=1}^{n} V_{j}\left(G_{i j} \cos \delta_{i j}+B_{i j} \sin \delta_{i j}\right) \\
Q_{i}=V_{i} \sum_{j=1}^{n} V_{j}\left(G_{i j} \sin \delta_{i j}-B_{i j} \cos \delta_{i j}\right) \\
P_{i j}=-t_{i j} G_{i j} V_{i}^{2}+V_{i} V_{j}\left(G_{i j} \cos \delta_{i j}+B_{i j} \sin \delta_{i j}\right) \\
Q_{i j}=t_{i j} B_{i j} V_{i}^{2}-0.5 B_{i j} V_{i}^{2}+V_{i} V_{j}\left(G_{i j} \sin \delta_{i j}-B_{i j} \cos \delta_{i j}\right)
\end{array}\right.
$$

where $P_{i}$ is the active power leaving bus $i, Q_{i}$ is the reactive power leaving bus $i, V_{i}$ and $\delta_{i}$ are the bus voltage magnitude and angle, respectively; $\delta_{i j}=\delta_{i}-\delta_{j} ; n$ is the number of lines connected to bus $i ; G_{i j}$ and $B_{i j}$ are the real and imaginary parts of the admittance of line $i j$, respectively; $P_{i j}$ and $Q_{i j}$ are the active and reactive power flow in line $i j$, respectively; $t_{i j}$ is the transformer tap ratio.

To describe the impact of wind power variation on system state variables such as line active and reactive flow and bus voltage magnitudes and angles, (1) is linearized around the system operating point. The system operating point may change after the generation dispatch operation, 
but it can be considered fixed at the time of the next generation dispatch. The resulting model is written as follows [3]:

$$
\left\{\begin{array}{l}
{\left[\begin{array}{c}
\Delta \boldsymbol{\delta} \\
\Delta \boldsymbol{V}
\end{array}\right]=\boldsymbol{G}^{-1}\left[\begin{array}{c}
\Delta \boldsymbol{P} \\
\Delta \boldsymbol{Q}
\end{array}\right]=\boldsymbol{K}\left[\begin{array}{c}
\Delta \boldsymbol{P} \\
\Delta \boldsymbol{Q}
\end{array}\right]} \\
{\left[\begin{array}{c}
\Delta \boldsymbol{P}_{\text {line }} \\
\Delta \boldsymbol{Q}_{\text {line }}
\end{array}\right]=\boldsymbol{H}\left[\begin{array}{c}
\Delta \boldsymbol{\delta} \\
\Delta \boldsymbol{V}
\end{array}\right]=\boldsymbol{H} \boldsymbol{K}\left[\begin{array}{c}
\Delta \boldsymbol{P} \\
\Delta \boldsymbol{Q}
\end{array}\right]=\boldsymbol{L}\left[\begin{array}{c}
\Delta \boldsymbol{P} \\
\Delta \boldsymbol{Q}
\end{array}\right]}
\end{array}\right.
$$

where all the variables are in vector or matrix form, and the symbol $\Delta$ indicates the variation of the variables, especially, $\Delta \boldsymbol{P}_{\text {line }}$ and $\Delta \boldsymbol{Q}_{\text {line }}$ are the variation of the active and reactive line power flow, respectively; $\boldsymbol{G}$ and $\boldsymbol{H}$ are the Jacobian matrices at the system operating point; $\boldsymbol{K}$ and $\boldsymbol{L}$ are the sensitivity matrices.

Separating components of (2) gives

$$
\left\{\begin{array}{l}
{\left[\begin{array}{c}
\Delta \boldsymbol{\delta} \\
\Delta \boldsymbol{V}
\end{array}\right]=\boldsymbol{K}\left[\begin{array}{c}
\Delta \boldsymbol{P} \\
\Delta \boldsymbol{Q}
\end{array}\right]=\left[\begin{array}{llll}
\boldsymbol{K}_{d} & \boldsymbol{K}_{w} & \boldsymbol{K}_{g} & \boldsymbol{K}_{Q}
\end{array}\right]\left[\begin{array}{c}
\Delta \boldsymbol{P}_{d} \\
\Delta \boldsymbol{P}_{w} \\
\Delta \boldsymbol{P}_{g} \\
\Delta \boldsymbol{Q}
\end{array}\right]} \\
{\left[\begin{array}{c}
\Delta \boldsymbol{P}_{\text {line }} \\
\Delta \boldsymbol{Q}_{\text {line }}
\end{array}\right]=\boldsymbol{L}\left[\begin{array}{c}
\Delta \boldsymbol{P} \\
\Delta \boldsymbol{Q}
\end{array}\right]=\left[\begin{array}{llll}
\boldsymbol{L}_{d} & \boldsymbol{L}_{w} & \boldsymbol{L}_{g} & \boldsymbol{L}_{Q}
\end{array}\right]\left[\begin{array}{c}
\Delta \boldsymbol{P}_{d} \\
\Delta \boldsymbol{P}_{w} \\
\Delta \boldsymbol{P}_{g} \\
\Delta \boldsymbol{Q}
\end{array}\right]}
\end{array}\right.
$$

where $\Delta \boldsymbol{P}_{d}$ and $\Delta \boldsymbol{P}_{w}$ are the variation of the load and wind power; $\Delta \boldsymbol{P}_{g}$ is the required adjustment of the conventional generators.

\subsection{Model for generation dispatch}

The variation of wind power and system load affects both the bus voltage and the line power flows. To maintain the stability of the power system, the conventional generators should be scheduled to balance the power supply and load.In this paper, the unit commitment problem is assumed to be solved in advance, and the model of generation dispatch is written as [3]:

$\Delta \boldsymbol{P}_{g}=\boldsymbol{T}\left[\begin{array}{c}\Delta \boldsymbol{P}_{w} \\ \mathbf{1}_{N_{d}}^{\mathrm{T}} \Delta \boldsymbol{P}_{d}\end{array}\right]=\left[\begin{array}{ll}\boldsymbol{T}_{w} & \boldsymbol{T}_{d}\end{array}\right]\left[\begin{array}{c}\Delta \boldsymbol{P}_{w} \\ \mathbf{1}_{N_{d}}^{\mathrm{T}} \Delta \boldsymbol{P}_{d}\end{array}\right]$

where $\boldsymbol{T}$ is the matrix of distribution weights of the wind power and system load fluctuation with respect to the conventional generators participating in dispatch. It represents the decision strategy for generation dispatch; each distribution weight $\boldsymbol{T}_{i j}$ in $\boldsymbol{T}$ indicates the adjustment of the $i^{\text {th }}$ generator output corresponding to unity change of the $j^{\text {th }}$ wind power output or system load. Matrix $\boldsymbol{T}_{w}$ and vector $\boldsymbol{T}_{d}$ contain all the elements corresponding to the variation of wind power and system load, respectively; $N_{d}$ is the number of load buses, and $\mathbf{1}_{N_{d}}$ is an $N_{d}$-dimension unit row vector; and each element $\Delta \boldsymbol{P}_{g, i}$ in the vector $\Delta \boldsymbol{P}_{g}$ indicates the adjustment of the $i^{\text {th }}$ generator output.

The generation dispatch model (4) adjusts the conventional generation to accommodate the variation of wind power and system load, and the adjustment of each generator depends on the dispatch strategy and the specific variation of wind power and system load.

Taking the generation dispatch into consideration, the linear power flow model can be rewritten as:

$$
\left\{\begin{aligned}
& {\left[\begin{array}{c}
\Delta \boldsymbol{\delta} \\
\Delta \boldsymbol{V}
\end{array}\right]=} {\left[\begin{array}{lll}
\left(\boldsymbol{K}_{d}+\boldsymbol{K}_{g} \boldsymbol{T}_{d} \cdot \mathbf{1}^{\mathrm{T}}\right) & \left(\boldsymbol{K}_{w}+\boldsymbol{K}_{g} \boldsymbol{T}_{w}\right) & \boldsymbol{K}_{Q}
\end{array}\right]\left[\begin{array}{c}
\Delta \boldsymbol{P}_{d} \\
\Delta \boldsymbol{P}_{w} \\
\Delta \boldsymbol{Q}
\end{array}\right] } \\
&= \boldsymbol{K}^{\prime}\left[\begin{array}{c}
\Delta \boldsymbol{P}_{d} \\
\Delta \boldsymbol{P}_{w} \\
\Delta \boldsymbol{Q}
\end{array}\right] \\
& {\left[\begin{array}{c}
\Delta \boldsymbol{P}_{\text {line }} \\
\Delta \boldsymbol{Q}_{\text {line }}
\end{array}\right]=\left[\begin{array}{lll}
\left(\boldsymbol{L}_{d}+\boldsymbol{L}_{g} \boldsymbol{T}_{d} \cdot \mathbf{1}^{\mathrm{T}}\right) & \left(\boldsymbol{L}_{w}+\boldsymbol{L}_{g} \boldsymbol{T}_{w}\right) & \boldsymbol{L}_{Q}
\end{array}\right]\left[\begin{array}{c}
\Delta \boldsymbol{P}_{d} \\
\Delta \boldsymbol{P}_{w} \\
\Delta \boldsymbol{Q}
\end{array}\right] } \\
&=\boldsymbol{L}^{\prime}\left[\begin{array}{c}
\Delta \boldsymbol{P}_{d} \\
\Delta \boldsymbol{P}_{w} \\
\Delta \boldsymbol{Q}
\end{array}\right]
\end{aligned}\right.
$$

One goal of this paper is to find the optimal generation dispatch strategy that minimizes the conventional generation cost of accommodating the uncertainty of wind power and system load. To achieve this goal, PPF analysis based on the principle of maximum entropy will be developed in Section 3, so as to establish a probabilistic circumstance that is the closest to the actual situation; after that, in Section 4, a chance-constrained programming model will be presented and solved to obtain the optimal generation dispatch strategy.

\section{PPF analysis based on the maximum entropy principle}

If the generation dispatch strategy is known, that is, the distribution matrix $\boldsymbol{T}$ is given, then partial information about the system state random variables, such as $\Delta \boldsymbol{P}_{\text {line }}$, can be obtained by (4), (5) and the probabilistic features of wind power and system load. Based on this partial information, in order to calculate the most likely realized probability distributions of the system state variables, the maximum entropy principle and the related cumulant calculation process are presented.

\subsection{The maximum entropy principle}

The maximum entropy principle was first proposed by E.T. Jaynes in [23] in these terms: "The maximum-entropy estimate is the least biased estimate possible on the given 
information; i.e., it is maximally noncommittal with regard to missing information", where the maximum entropy estimate criterion is called the principle of maximum entropy.

According to [23], the principle of maximum entropy can be applied to find the distribution estimate that is the closest to the real distribution, that is, the most likely realized probability distribution. The following maximum entropy model [24] is applied for this purpose:

$$
\begin{cases}\max _{f(\Delta P)} & h(\Delta P)=-\int f(\Delta P) \ln f(\Delta P) \mathrm{d} \Delta P \\ \text { s.t. } & E\left[\Delta P^{n}\right]=\int \Delta P^{n} f(\Delta P) \mathrm{d} \Delta P=\mu_{n} \\ & \int f(\Delta P) \mathrm{d} \Delta P=1\end{cases}
$$

where $\Delta P$ is the random variable of interest, about which partial information is given by the constraints of moments; $h(\Delta P)$ is the entropy of $\Delta P ; f(\Delta P)$ is the PDF of $\Delta P ; \mu_{n}$, $n=1,2, \ldots, N$ are the moments of $f(\Delta P)$.

\subsection{The cumulant calculation process}

In the maximum entropy model, the moments $\mu_{n}, n=1$, $2, \ldots, N$ of the random variable need to be provided. The moments can be obtained conveniently from the corresponding cumulants of the random variable [25]:

$$
\left\{\begin{array}{l}
\mu_{1}=\kappa_{1} \\
\mu_{2}=\kappa_{2}+\kappa_{1}^{2} \\
\mu_{3}=\kappa_{3}+3 \kappa_{2} \kappa_{1}+\kappa_{1}^{3} \\
\mu_{4}=\kappa_{4}+4 \kappa_{3} \kappa_{1}+3 \kappa_{2}^{2}+6 \kappa_{2} \kappa_{1}^{2}+\kappa_{1}^{4} \\
\mu_{5}=\kappa_{5}+5 \kappa_{4} \kappa_{1}+10 \kappa_{3} \kappa_{2}+10 \kappa_{3} \kappa_{1}^{2}+15 \kappa_{2}^{2} \kappa_{1}+10 \kappa_{2} \kappa_{1}^{3}+\kappa_{1}^{5} \\
\cdots
\end{array}\right.
$$

where $\kappa_{v}$ is the $v$ th cumulant of the random variable. The cumulants can also be obtained from the moments:

$$
\left\{\begin{aligned}
\kappa_{1}= & \mu_{1} \\
\kappa_{2}= & \mu_{2}-\mu_{1}^{2} \\
\kappa_{3}= & \mu_{3}-3 \mu_{2} \mu_{1}+2 \mu_{1}^{3} \\
\kappa_{4}= & \mu_{4}-4 \mu_{3} \mu_{1}-3 \mu_{2}^{2}+12 \mu_{2} \mu_{1}^{2}-6 \mu_{1}^{4} \\
\kappa_{5}= & \mu_{5}-5 \mu_{4} \mu_{1}-10 \mu_{3} \mu_{2}+20 \mu_{3} \mu_{1}^{2}+30 \mu_{2}^{2} \mu_{1}-60 \mu_{2} \mu_{1}^{3} \\
& +24 \mu_{1}^{5} \\
\ldots &
\end{aligned}\right.
$$

In order to obtain the input moments, some theoretical analysis is needed in order to calculate the cumulants. If a random variable $\eta$ is a linear function of $N$ random variables $\quad \theta_{i}, \quad i=1,2, \ldots, m, \quad$ that is, $\eta=a_{0}+a_{1} \theta_{1}+\cdots+a_{m} \theta_{m}$, then once the cumulants of $\theta_{i}, i=1,2, \ldots, m$ are known, the cumulants of $\eta$ can be calculated by these equations [26]:

$$
\left\{\begin{array}{l}
\kappa_{\eta, 1}=a_{0}+\sum_{i=1}^{m} a_{i} \kappa_{\theta_{i}, 1} \\
\kappa_{\eta, 2}=\sum_{i=1}^{m} a_{i}^{2} \kappa_{\theta_{i}, 2}+2 \sum_{i=1, i<j}^{m} a_{i} a_{j} \kappa_{\theta_{i}, \theta_{j}} \\
\kappa_{\eta, 3}=\sum_{i=1}^{m} a_{i}^{3} \kappa_{\theta_{i}, 3}+3 \sum_{i=1, i \neq j}^{m} a_{i}^{2} a_{j} \kappa_{\theta_{i}^{2}, \theta_{j}}+6 \sum_{i=1, i<j<k}^{m} a_{i} a_{j} a_{k} \kappa_{\theta_{i}, \theta_{j}, \theta_{k}} \\
\ldots
\end{array}\right.
$$

where $\kappa_{\eta, v}$ is the $v$ th cumulant of $\eta ; \kappa_{\theta_{i}, v}, i=1,2, \ldots, m$ is the $v$ th cumulant of $\theta_{i} ; \kappa_{\theta_{i}, \theta_{j}}, \kappa_{\theta_{i}^{2}, \theta_{j}}$ and $\kappa_{\theta_{i}, \theta_{j}, \theta_{k}}$ are the joint cumulants of the random variables, which are introduced in [26].

If $\theta_{1}, \theta_{2}, \ldots, \theta_{m}$ are independent, then (9) can be simplified to

$\left\{\begin{array}{l}\kappa_{\eta, 1}=a_{0}+a_{1} \kappa_{\theta_{1}, 1}+\cdots+a_{m} \kappa_{\theta_{m}, 1} \quad v=1 \\ \kappa_{\eta, v}=a_{1}^{v} \kappa_{\theta_{1}, v}+\cdots+a_{m}^{v} \kappa_{\theta_{m}, v} \quad v \geq 2\end{array}\right.$

\subsection{Probabilistic power flow analysis}

To solve the most likely realized probability distribution of $\Delta \boldsymbol{P}_{\text {line }}$, firstly, the moments of the uncertainty sources, wind power and system load, can be collected by statistical techniques from the historical data, and converted to cumulants. Secondly, the cumulants of $\Delta \boldsymbol{P}_{\text {line }}$ are calculated according to (5) and (9), and converted to moments. Thirdly, the moments of $\Delta \boldsymbol{P}_{\text {line }}$ are input into the maximum entropy model (6).

The general solution of the maximum entropy model has been proposed in [27],

$f(\Delta P)=\exp \left[-\sum_{n=0}^{N} \lambda_{n} \Delta P^{n}\right]$

where $\lambda_{n}=\left[\lambda_{0}, \lambda_{1}, \ldots, \lambda_{N}\right]$ are Lagrangian multipliers.

Substituting (11) into the optimization model of (6) results in these equations:

$\left\{\begin{array}{l}\int \Delta P^{n} \exp \left[-\sum_{n=0}^{N} \lambda_{n} \Delta P^{n}\right] \mathrm{d} \Delta P=\mu_{n} \\ \int \exp \left[-\sum_{n=0}^{N} \lambda_{n} \Delta P^{n}\right] \mathrm{d} \Delta P=1\end{array}\right.$

To solve the values of Lagrangian multipliers $\lambda_{n}=\left[\lambda_{0}\right.$, $\left.\lambda_{1}, \ldots, \lambda_{N}\right]$, the Newton iteration method can be applied as in [27]. Equation (12) can be rewritten as: 
$\Psi_{n}(\boldsymbol{\lambda})=\int \Delta P^{n} \exp \left[-\sum_{n=0}^{N} \lambda_{n} \Delta P^{n}\right] \mathrm{d} \Delta P=\mu_{n}$

where $\mu_{0}=1$. Using trial initial vector $\lambda^{0}, \Psi_{n}(\lambda)$ can be developed as a first order Taylor's series around $\lambda^{0}$ :

$$
\Psi_{n}(\lambda) \cong \Psi_{n}\left(\lambda^{0}\right)+\left(\lambda-\lambda^{0}\right)^{\mathrm{T}}\left[\operatorname{grad} \Psi_{n}(\lambda)\right]_{\left(\lambda=\lambda^{0}\right)}=\mu_{n}
$$

By denoting

$\zeta=\lambda-\lambda^{0}$

$\gamma=\left[\mu_{0}-\Psi_{0}\left(\lambda^{0}\right), \ldots, \mu_{N}-\Psi_{N}\left(\lambda^{0}\right)\right]^{\mathrm{T}}$

$\boldsymbol{\Psi}=\left[\psi_{n k}\right]=\left[\frac{\partial \Psi_{n}(\boldsymbol{\lambda})}{\partial \lambda_{k}}\right]_{\left(\lambda=\lambda^{0}\right)} n, k=0,1, \ldots, N$

(14) can be abbreviated as:

$\Psi \zeta=\gamma$

Thus $\zeta$ can be solved from (18), and $\lambda=\lambda^{0}+\zeta$ becomes the new initial vector which is input to (15)-(18) again. The iterations continue until $\zeta$ becomes small enough, thus the Lagrangian multipliers $\lambda_{n}=\left[\lambda_{0}, \lambda_{1}, \ldots, \lambda_{N}\right]$ are solved.

The most likely realized probability distributions of $\Delta \boldsymbol{\delta}$, $\Delta \boldsymbol{V}$ and $\Delta \boldsymbol{Q}_{\text {line }}$ can all be solved in this way.

\section{Chance-constrained optimization model for the optimal generation dispatch strategy}

In this section, a chance-constrained optimization model is presented to determine the optimal generation dispatch strategy that minimizes the generation cost. The solution algorithm is also presented using the most likely realized probability distributions of the system state variables obtained in the previous section.

\subsection{Chance-constrained optimization model for generation dispatch strategy}

The optimal generation dispatch strategy should minimize the expected generation cost, while avoiding overload of each system branch at a certain probability. Thus, the generation dispatch problem can be formulated as the following chance-constrained programming model.

$$
\begin{array}{ll}
\min _{T} & E\left[\mathbf{1}^{\mathrm{T}} \boldsymbol{C}\left(\boldsymbol{P}_{g, 0}+\Delta \boldsymbol{P}_{g}\right)\right] \\
\text { s.t. } & \operatorname{Pr}\left(\left|\boldsymbol{P}_{l, 0}+\Delta \boldsymbol{P}_{\text {line }}\right| \leq \boldsymbol{P}_{l, \text { max }}\right) \geq \alpha
\end{array}
$$

$\left[\begin{array}{c}\Delta \boldsymbol{P}_{\text {line }} \\ \Delta \boldsymbol{Q}_{\text {line }}\end{array}\right]=\boldsymbol{L}^{\prime}\left[\begin{array}{c}\Delta \boldsymbol{P}_{d} \\ \Delta \boldsymbol{P}_{w} \\ \Delta \boldsymbol{Q}\end{array}\right]$

$\Delta \boldsymbol{P}_{g}=\boldsymbol{T}\left[\begin{array}{c}\Delta \boldsymbol{P}_{w} \\ \mathbf{1}^{\mathrm{T}} \Delta \boldsymbol{P}_{d}\end{array}\right]=\left[\begin{array}{ll}\boldsymbol{T}_{w} & \boldsymbol{T}_{d}\end{array}\right]\left[\begin{array}{c}\Delta \boldsymbol{P}_{w} \\ \mathbf{1}_{N_{d}}^{\mathrm{T}} \Delta \boldsymbol{P}_{d}\end{array}\right]$

$\boldsymbol{P}_{g, \min } \leq \boldsymbol{P}_{g, 0}+\Delta \boldsymbol{P}_{g} \leq \boldsymbol{P}_{g, \max }$

$-1 \leq T_{w, i j} \leq 0$

$\sum_{i=1}^{N_{g}} T_{w . i j}=-1 \quad j=1,2, \ldots, N_{w}$

$0 \leq T_{d, i} \leq 1$

$\sum_{i=1}^{N_{g}} T_{d, i}=1$

$\left\{\begin{array}{l}i=1,2, \ldots, N_{g} \\ j=1,2, \ldots, N_{w}\end{array}\right.$

where the decision variable $\boldsymbol{T}$ is defined above in Section 2.2; $\boldsymbol{P}_{g, 0}$ is the conventional generation output at the system operation point; $\boldsymbol{C}\left(\boldsymbol{P}_{g, 0}+\Delta \boldsymbol{P}_{g}\right)$ is the vector of generation cost, each element

$C_{i}\left(P_{g, 0}^{i}+\Delta P_{g}^{i}\right)=a_{i}\left(P_{g, 0}^{i}+\Delta P_{g}^{i}\right)^{2}+b_{i}\left(P_{g, 0}^{i}+\Delta P_{g}^{i}\right)+c_{i}$

$E[\cdot]$ denotes the calculation of expected value, and $\operatorname{Pr}(\cdot)$ denotes the probability; $\boldsymbol{P}_{l, 0}$ is the vector of line active power flow at the system operating point; $\boldsymbol{P}_{g, \text { min }}$ and $\boldsymbol{P}_{g, \text { max }}$ are the minimum and maximum generation limits; $T_{w, i j}$ is the element of $\boldsymbol{T}_{w} ; T_{d, i}$ is the element of $\boldsymbol{T}_{d} ; N_{g}$ is the number of dispatchable conventional generators; $N_{w}$ is the number of wind farms that connected to the power grid.

In this model, the object is to minimize the expected generation cost. The chance constraint (20) indicates that the probability of not overloading each line should be no smaller than $\alpha$. Equation (23) is the constraint of conventional generation output limits. Equations (24)-(27) indicate that the variations of wind power and system load are compensated by the dispatchable conventional generators, and allocated to each generator according to the dispatch strategy.

The chance-constrained programming model (19)-(28) is difficult to solve because of the chance constraint (20). In the next subsection, the pattern search algorithm combined with the maximum entropy method will be proposed to solve it and obtain the optimal generation dispatch strategy.

\subsection{Solution algorithm}

As discussed in Section 3, if the generation dispatch strategy $\boldsymbol{T}$ is given, then the most likely realized probability 
distribution estimates of $\Delta \boldsymbol{P}_{\text {line }}, \Delta \boldsymbol{Q}_{\text {line }}, \Delta \boldsymbol{\delta}$ and $\Delta \boldsymbol{V}$ can be solved by the cumulant calculation process and the maximum entropy model. Once the PDF of $\Delta \boldsymbol{P}_{\text {line }}$ is known, the probability of not overloading each line is calculated as:

$\operatorname{Pr}\left(\left|P_{l, 0}^{i}+\Delta P_{\text {line }}^{i}\right| \leq P_{l, \text { max }}^{i}\right)=\int_{-P_{l, \text { max }}^{i}-P_{l, 0}^{i}}^{P_{l, \text { max }}^{i}-P_{l, 0}^{i}} f\left(\Delta P_{\text {line }}^{i}\right) \mathrm{d} \Delta P_{\text {line }}^{i}$

where $i=1,2, \ldots, N_{l}$ indicates the $i^{\text {th }}$ line.

To obtain the optimal generation dispatch strategy, the computation procedure that combines the pattern search algorithm [28] with the maximum entropy method is shown as a flow chart in Fig. 1.

\section{Case study}

The maximum entropy-based generation dispatching method has been tested on the benchmark IEEE-39 bus power system consisting of 10 conventional generators. To simplify the analysis, the output of generators at buses 32 , $34,36,37,39$ is fixed to be the output at the operating point. The generators at buses $30,31,33,35,38$ respond to generation dispatching, balancing the variation of wind power and system load.

The wind farms are assumed to be independent of each other, and connected to bus 24 , bus 25 and bus 29 , respectively. Because of the lack of historical data, pseudorandom wind speed data are generated from known Weibull distributions, and the wind power penetration considered is $10 \%$ of the total load at the operating point. The active power loads are assumed to be independent, and historical load data are generated from normal distributions, the expected values of which are set to be the load value at the operating point. The confidence level of not overloading each branch is set to be $95 \%$.

To validate the effectiveness of the optimal dispatching strategy, that is, reducing the generation cost and accommodating the fluctuation of wind power and system load, tests are carried out with and without the optimal dispatching strategy. The generation output at the slack bus (bus 31) entirely balances the power uncertainties when the optimal dispatching strategy is not used.

To validate the effectiveness of the maximum entropybased (ME) method, the Gram-Charlier (GC) [11] and Monte Carlo (MC) [7] methods are also applied to the same power system to do the PPF analysis. The GC method calculates the cumulants of the system state random variables exactly the same way as the ME method, but obtains their PDFs by GC expansion formulations. The MC method enumerates all the historical wind power and system load data, and calculates corresponding sample values

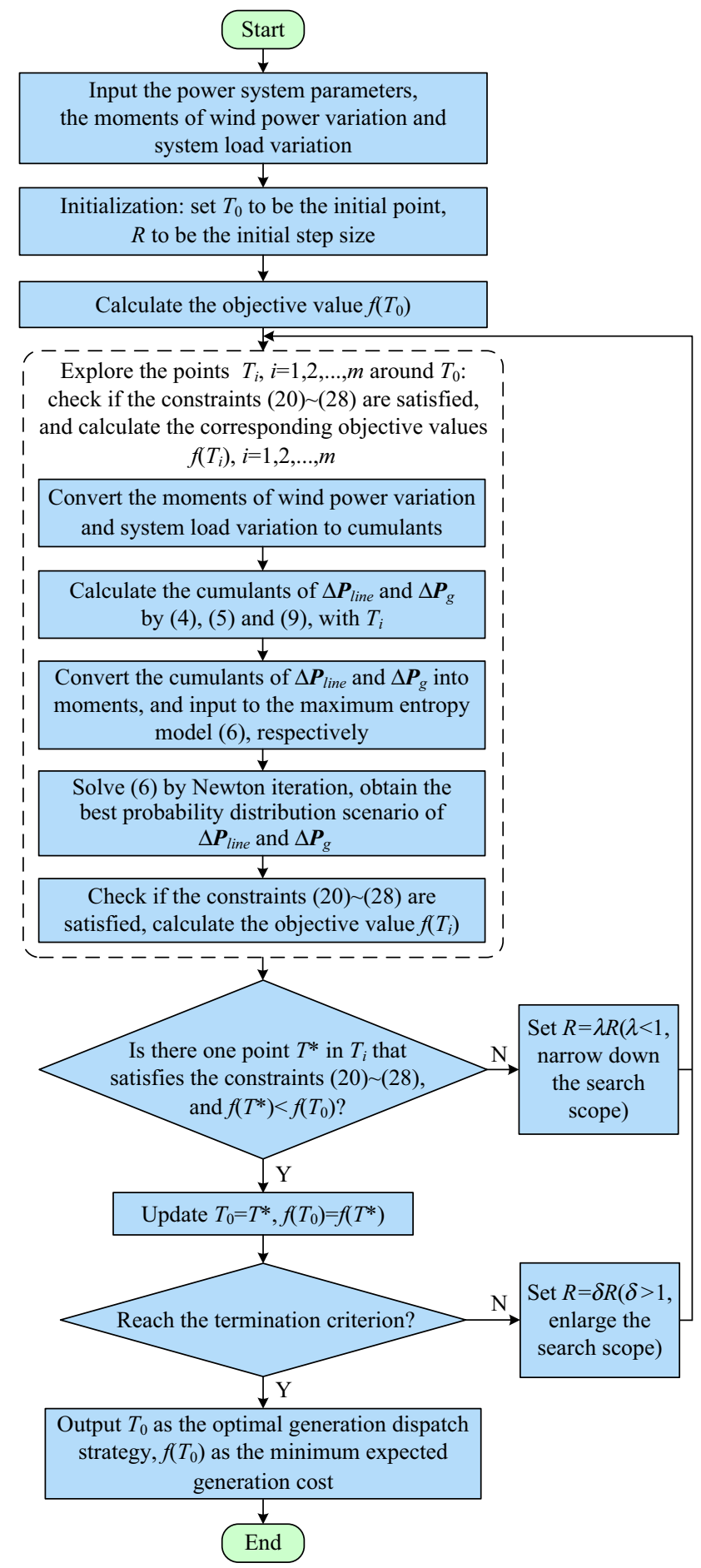

Fig. 1 Flow chart of the pattern search algorithm combined with the maximum entropy method

of the system state random variables, so as to evaluate their PDFs. The details of the GC method and the MC method can be found in $[7,11]$ respectively.

The results of MC algorithm are used as a benchmark, and the accuracies of the maximum entropy-based method and the GC algorithm are evaluated by the average root 
mean square (ARMS) error [3], which is formulated as follows:

$A R M S=\frac{\sqrt{\sum_{i=1}^{N}\left(Y_{i}-Y_{r e f, i}\right)^{2}}}{N}$

where $Y_{i}$ is the $i^{\text {th }} \mathrm{CDF}$ value of the random variable obtained by the evaluated algorithm, and $Y_{r e f, i}$ is the corresponding $\mathrm{CDF}$ value obtained by the $\mathrm{MC}$ algorithm; $N$ is the total number of evaluated points.

\subsection{Expected cost with and without the optimal generation dispatching strategy}

The optimal generation dispatching strategy is obtained by solving the chance-constrained programming model (19)-(28), where the object is to minimize the expected value of total generation cost, and the generation dispatching strategy is denoted by the distribution matrix $\boldsymbol{T}$. Table 1 lists the expected value of generation cost with and without the optimal generation dispatch strategy. The results show that with the optimal generation dispatch strategy, the expected generation cost decreases. The main reason is that the more economic conventional generators share the burden of balancing the power mismatch with the generator at the slack bus, thus decreasing the cost.

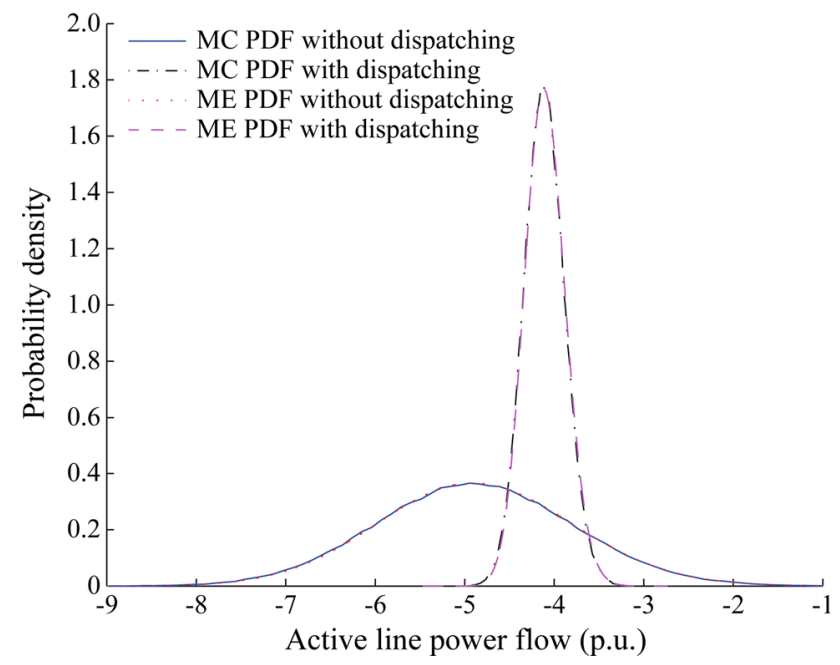

Fig. 2 PDF curves of the active line flow on branch 5-6 obtained by ME method and MC method with and without generation dispatching

\subsection{Probabilistic power flow analysis of bus voltage angle and active line flow}

In this study, the generation dispatch strategy mainly affects the active line flow and the bus voltage angle, so the PPF analysis has been performed on the IEEE-39 bus system to illustrate this effect. Figure 2 shows the PDF curves of the active line flow on branch 5-6 obtained by the ME method and the MC method, with and without generation dispatching; Table 2 lists the ARMS error, mean value, standard deviation, $10 \%$ confidence level, $90 \%$

Table 1 Comparison of the expected generation cost with and without generation dispatching

\begin{tabular}{lll}
\hline Parameter & Without dispatching & With dispatching \\
\hline Expected value of generation cost (\$) & 41172.2 & 40567.6 \\
Computation time (s) & 6 & 511 \\
\hline
\end{tabular}

Table 2 Comparison of results for the active line flow on branch 5-6 obtained by different methods with and without generation dispatch

\begin{tabular}{|c|c|c|c|c|c|c|}
\hline \multirow[t]{2}{*}{ Index } & \multicolumn{3}{|c|}{ Without dispatching } & \multicolumn{3}{|c|}{ With dispatching } \\
\hline & $\mathrm{MC}$ & $\mathrm{GC}$ & $\mathrm{ME}$ & $\mathrm{MC}$ & $\mathrm{GC}$ & ME \\
\hline ARMS (\%) & 0 & 0.00273 & 0.00272 & 0 & 0.00282 & 0.00199 \\
\hline Mean (p.u.) & -4.87021 & -4.87020 & -4.87021 & -4.10102 & -4.10102 & -4.10102 \\
\hline Std (p.u.) & 1.09578 & 1.09455 & 1.09455 & 0.22811 & 0.22785 & 0.22785 \\
\hline 10\%CL (p.u.) & -6.27261 & -6.26357 & -6.26352 & -4.38871 & -4.38902 & -4.38909 \\
\hline 90\%CL (p.u.) & -3.46411 & -3.46042 & -3.46052 & -3.80663 & -3.80687 & -3.80709 \\
\hline Computation time (s) & 2223.8 & 36.5 & 5.8 & 2316.5 & 38.5 & 3.0 \\
\hline
\end{tabular}




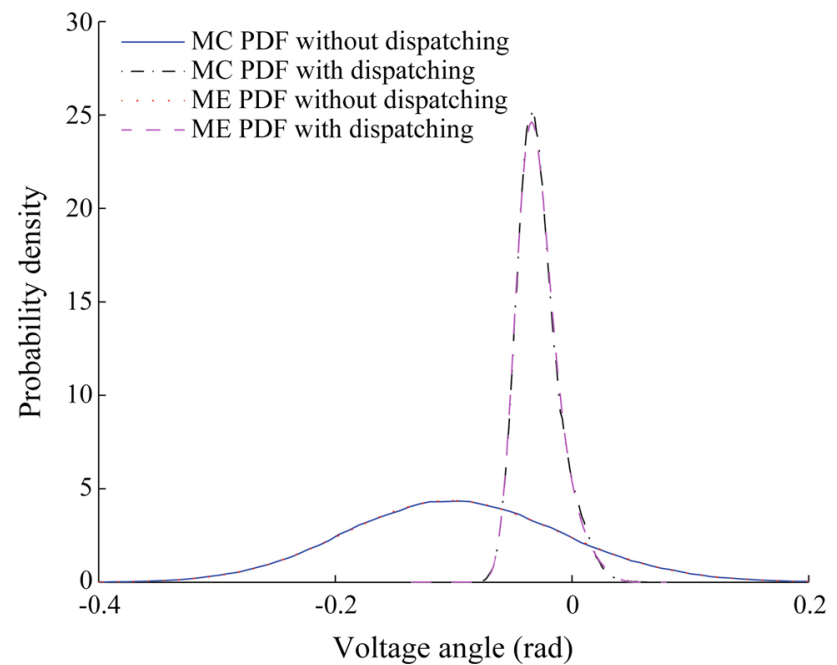

Fig. 3 PDF curves of the voltage angle at bus 25 obtained by the ME method and the MC method with and without generation dispatching

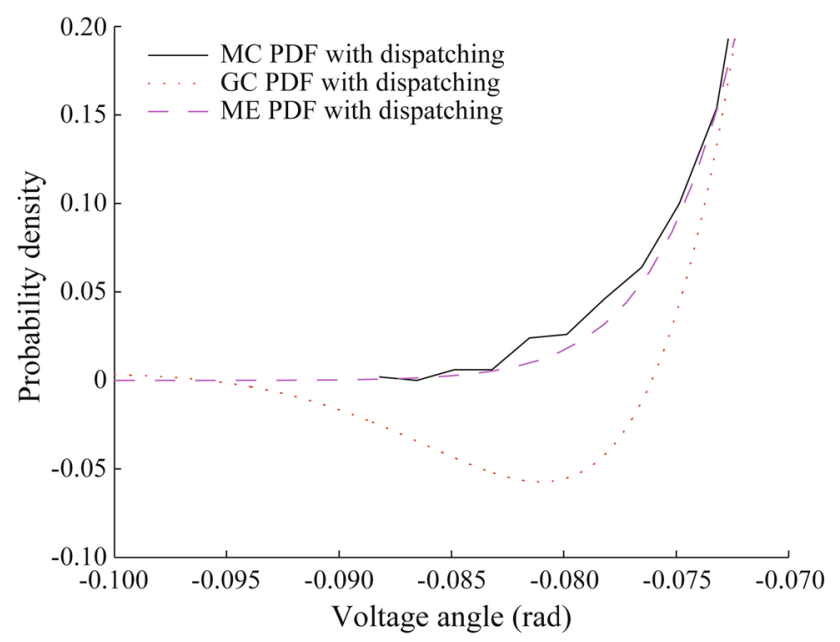

Fig. 4 Part of the PDF curves of the voltage angle at bus 25 obtained by different methods

confidence level and the computation time of the PPF results obtained by the ME method, the GC method and the MC method, with and without generation dispatching.
Figure 3 shows the PDF curves of the voltage angle at bus 25 obtained by the ME method and the MC method, while Fig. 4 shows part of the PDF curves of the voltage angle at bus 25 obtained by the ME method, the GC method and the MC method. Table 3 lists the statistical parameters of the voltage angle results obtained by the ME method, the GC method and the MC method, with and without generation dispatching.

From Table 2, it can be seen that, compared with the results of MC method, the ME method requires less computation time while obtaining accurate PDF curves of the active line power flow and the bus voltage angle. Though the GC method also computes much faster than the MC method, the PDF curve obtained by GC method may have negative probability density values, as shown in Fig. 4, which is unrealistic.

Comparing the PDF curves and the results obtained with and without generation dispatching, it can be seen that the dispatch strategy affects the probabilistic active power flow and bus voltage angle significantly. In most cases, the generation dispatching reduces the variation of both active power flow and bus voltage angle. The main reason is that multiple conventional generators distributed across the power grid, rather than only the generator at the slack bus, participate in compensating the variation of wind power and system load, so as to decrease the variation of power flow. However, in some cases, generally when the bus or the branch is near a conventional generator participating in dispatching, the uncertainty of the bus voltage angle or the active line flow increases. For example, the PDF curves of the active power flow on line 21-22 is shown in Fig. 5. The results show that the variation of the active power flow increases with generation dispatching, and this line is near the generator at bus 35 .

\subsection{Probabilistic power flow analysis of slack bus generation}

Without generation dispatching, the generation at the slack bus is responsible for balancing the total power

Table 3 Comparison of results for the voltage angle at bus 25 obtained by different methods with and without generation dispatch

\begin{tabular}{|c|c|c|c|c|c|c|}
\hline \multirow[t]{2}{*}{ Index } & \multicolumn{3}{|c|}{ Without dispatching } & \multicolumn{3}{|c|}{ With dispatching } \\
\hline & $\mathrm{MC}$ & GC & ME & $\mathrm{MC}$ & GC & $\mathrm{ME}$ \\
\hline ARMS (\%) & 0 & 0.00273 & 0.00194 & 0 & 0.00963 & 0.01561 \\
\hline Mean (p.u.) & -0.09727 & -0.09727 & -0.09727 & -0.02811 & -0.02811 & -0.02811 \\
\hline Std (p.u.) & 0.09205 & 0.09196 & 0.09196 & 0.01801 & 0.01801 & 0.01801 \\
\hline 10\%CL (p.u.) & -0.21431 & -0.21379 & -0.21405 & -0.04892 & -0.04928 & -0.04918 \\
\hline 90\%CL (p.u.) & 0.02114 & 0.02145 & 0.02095 & -0.00354 & -0.00398 & -0.00418 \\
\hline
\end{tabular}




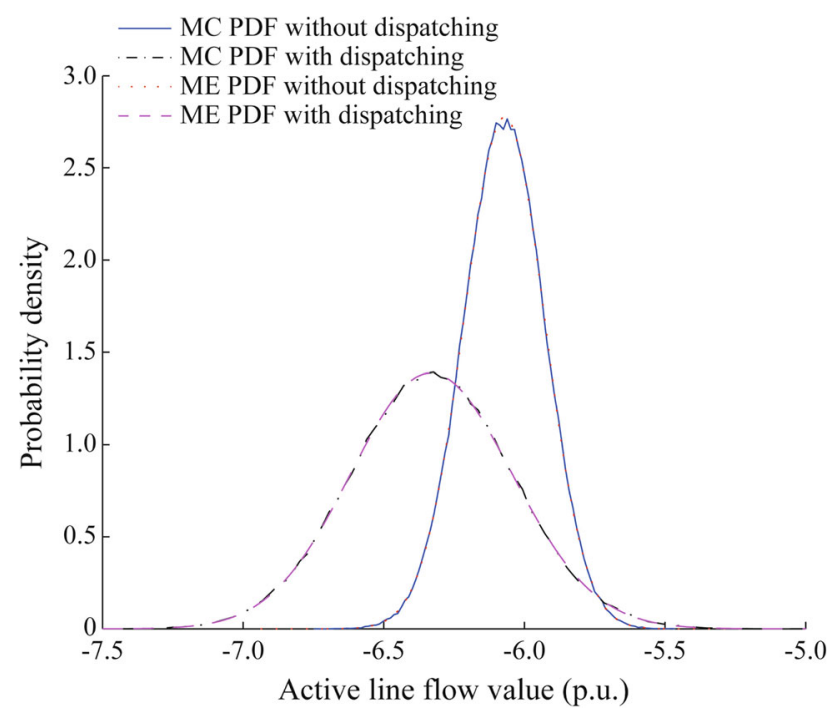

Fig. 5 PDF curves of the active line flow on branch 21-22 obtained by $\mathrm{ME}$ method and MC method with and without generation dispatching

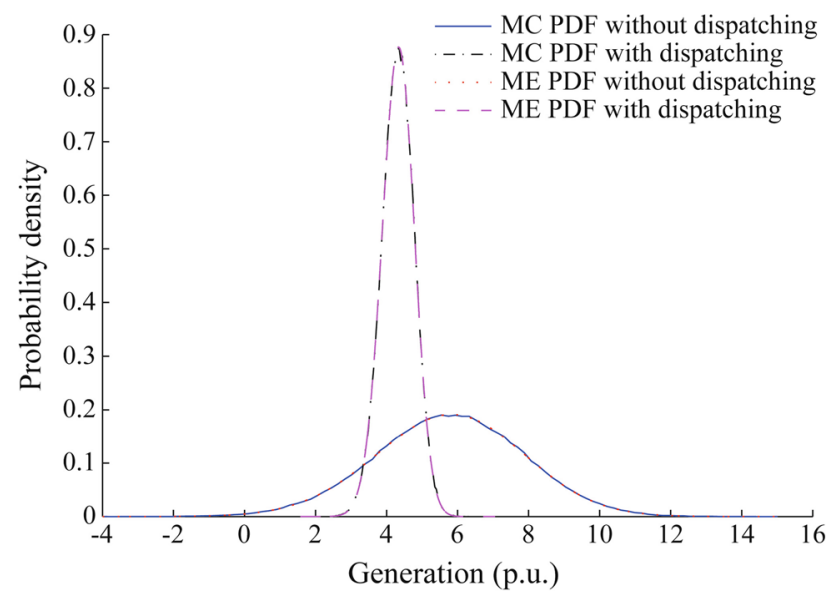

Fig. 6 PDF curves of active power generation at the slack bus obtained by ME method and MC method with and without generation dispatching mismatch in the power systems. With dispatching, several other conventional generators share this burden. The PDF curve of the generation at the slack bus are shown in Fig. 6, Table 4 lists the variation characteristics of generation at the slack bus. The results show that with generation dispatching, the variation of the generation at the slack bus decreases, because other conventional generators share in balancing the power mismatch.

\section{Conclusion}

In this paper, a new generation dispatch method based on the maximum entropy principle is presented to compensate the variation of wind power and system load, and to minimize the expected generation cost. With the available partial information about the statistics of uncertain factors, the ME principle is introduced to find the most likely realized probability distribution of the power flow, thus providing an accurate probabilistic power flow analysis which is necessary to solve the generation dispatching problem.

The proposed method is applied to the IEEE 39-bus system, and compared with MC and GC methods. The results demonstrate that the optimal generation dispatching strategy reduces the influence of uncertain factors on the system power flow, and decreases the expected generation costs. In addition, the results show that the ME method is superior to the GC method in accuracy, and is superior to the MC method in computational efficiency.

The ME-based method could also be applied to other problems of system planning and operation, such as transmission network planning and reserve scheduling, and could also be applied to deal with different types of uncertainties, such as the variability of photovoltaic generation.

Table 4 Comparison of results for generation at the slack bus obtained by different methods with and without generation dispatch

\begin{tabular}{|c|c|c|c|c|c|c|}
\hline \multirow[t]{2}{*}{ Index } & \multicolumn{3}{|c|}{ Without dispatching } & \multicolumn{3}{|c|}{ With dispatching } \\
\hline & $\mathrm{MC}$ & GC & ME & $\mathrm{MC}$ & GC & ME \\
\hline ARMS (\%) & 0 & 0.00230 & 0.00242 & 0 & 0.00234 & 0.00206 \\
\hline Mean (p.u.) & 5.75678 & 5.75677 & 5.75677 & 4.31639 & 4.31639 & 4.31639 \\
\hline Std (p.u.) & 2.09037 & 2.08823 & 2.08823 & 0.45736 & 0.45691 & 0.45692 \\
\hline 10\%CL (p.u.) & 3.06955 & 3.06713 & 3.06640 & 3.72506 & 3.72697 & 3.72590 \\
\hline 90\%CL (p.u.) & 8.41431 & 8.41508 & 8.41540 & 4.89447 & 4.89576 & 4.89590 \\
\hline
\end{tabular}


Open Access This article is distributed under the terms of the Creative Commons Attribution 4.0 International License (http:// creativecommons.org/licenses/by/4.0/), which permits unrestricted use, distribution, and reproduction in any medium, provided you give appropriate credit to the original author(s) and the source, provide a link to the Creative Commons license, and indicate if changes were made.

\section{References}

[1] Vrakopoulou M, Margellos K, Lygeros J et al (2013) A probabilistic framework for reserve scheduling and $N-1$ security assessment of systems with high wind power penetration. IEEE Trans Power Syst 28(4):3885-3896

[2] Yuan X (2013) Overview of problems in large-scale wind integrations. J Mod Power Syst Clean Energy 1(1):22-25

[3] Fan M, Vittal V, Heydt GT et al (2013) Probabilistic power flow analysis with generation dispatch including photovoltaic resources. IEEE Trans Power Syst 28(2):1797-1805

[4] Wang J, Zobba AF, Huang C et al (2016) Day-ahead allocation of operation reserve in composite power systems with large-scale centralized wind farms. J Mod Power Syst Clean Energy 4(2):238-247

[5] Liu X, Xu Z, Wong KP (2013) Recent advancement on technical requirements for grid integration of wind power. $\mathrm{J}$ Mod Power Syst Clean Energy 1(3):216-222

[6] Bofinger S, Luig A, Beyer HG (2002) Qualification of wind power forecasts. In: Proceedings of global wind power conference, Paris, France, 2002, 5 pp

[7] Lyu JK, Heo JH, Kim MK et al (2013) Impacts of wind power integration on generation dispatch in power systems. J Electr Eng Technol 8(3):453-463

[8] Hargreaves JJ, Hobbs BF (2012) Commitment and dispatch with uncertain wind generation by dynamic programming. IEEE Trans Sustain Energy 3(4):724-734

[9] Vicente WCB, Caire R, Hadjsaid N (2012) Probabilistic load flow for voltage assessment in radial systems with wind power. Int J Electr Power Energy Syst 41(1):27-33

[10] Yuan Y, Zhou J, Ju P et al (2011) Probabilistic load flow computation of a power system containing wind farms using the method of combined cumulants and Gram-Charlier expansion. IET Renew Power Gener 5(6):448-454

[11] Zhang P, Lee ST (2004) Probabilistic load flow computation using the method of combined cumulants and Gram-Charlier expansion. IEEE Trans Power Syst 19(1):676-682

[12] Usaola J (2009) Probabilistic load flow with wind production uncertainty using cumulants and Cornish-Fisher expansion. Int $\mathbf{J}$ Electr Power Energy Syst 31(9):474-481

[13] Yao S, Wang Y (2011) Cornish-fisher expansion for probabilistic power flow of the distribution system with wind energy system. In: Proceedings of 4 th international conference on electric utility deregulation and restructuring and power technologies (DRPT), Weihai, China, 6-9 July 2011, 6 pp

[14] Ruiz-Rodriguez FJ, Hernández JC, Jurado F (2012) Probabilistic load flow for photovoltaic distributed generation using the Cornish-Fisher expansion. Electr Power Syst Res 89:129-138

[15] Su CL (2005) Probabilistic load-flow computation using point estimate method. IEEE Trans Power Syst 20(4):1843-1851

[16] Verbic G, Canizares CA (2006) Probabilistic optimal power flow in electricity markets based on a twopoint estimate method. IEEE Trans Power Syst 21(4):1883-1893

[17] Morales JM, Pérez-Ruiz J (2007) Point estimate schemes to solve the probabilistic power flow. IEEE Trans Power Syst 22(4):1594-1601

[18] Allan RN, Silva AMLD, Burchett RC (1981) Evaluation methods and accuracy in probabilistic load flow solutions. IEEE Trans Power Appar Syst PAS-100(5):2539-2546
[19] Hatziargyriou ND, Karakatsanis TS, Papadopoulos M (1993) Probabilistic load flow in distribution systems containing dispersed wind power generation. IEEE Trans Power Syst 8(1):159-165

[20] Jiang Y, Chen X, Yu K et al (2017) Short-term wind power forecasting using hybrid method based on enhanced boosting algorithm. J Mod Power Syst Clean Energy 5(1):126-133

[21] $\mathrm{Hu}$ W, Min Y, Zhou Y et al (2017) Wind power forecasting errors modelling approach considering temporal and spatial dependence. J Mod Power Syst Clean Energy 5(3):489-498

[22] Ander GJ (1990) Probability concepts in electric power systems. Wiley, New York

[23] Jaynes ET (1957) Information theory and statistical mechanics. Phys Rev 106(4):620-630

[24] Williams T, Crawford C (2013) Probabilistic load flow modeling comparing maximum entropy and Gram-Charlier probability density function reconstructions. IEEE Trans Power Syst 28(1):272-280

[25] Bian Q, Xu Q, Sun L et al (2014) Grid-connected wind power capacity optimization based on the principle of maximum entropy. In: Proceedings of 2014 IEEE PES general meeting, National Harbor, USA, 27-31 July 2014, 5 pp

[26] Fan M, Vittal V, Heydt GT et al (2012) Probabilistic power flow studies for transmission systems with photovoltaic generation using cumulants. IEEE Trans Power Syst 27(4):2251-2261

[27] Mohammad-Djafari A (2001) A matlab program to calculate the maximum entropy distributions. https://doi.org/10.1007/978-94017-2219-3_16

[28] Torczon V (1997) On the convergence of pattern search algorithms. SIAM J Optim 7(1):1-25

Qiaoyan BIAN received her B.E. and Ph.D. degrees in electrical engineering from Zhejiang University, Hangzhou, China, in 2008 and 2015, respectively. From March 2010 to April 2013, she was a training jointly doctoral student with the Electrical and Computer Engineering Department, the University of Oklahoma, Norman, OK, USA. Her research interests include power system operation and planning, renewable energy integration, ancillary services and power system relay protection.

Yutao QIU received the M.S. degree in electrical engineering from North China Electric Power University, Baoding, China. His research interests include power system relay protection management and smart substation study.

Wenlian WU is currently a senior engineer in Hangzhou Power Supply Company of State Grid Zhejiang Electric Power Company. His research interests include power system relay protection and automation in substations.

Huanhai XIN received the Ph.D. degree in electrical engineering from Zhejiang University, Hangzhou, China, in 2007. He is currently a professor in the Department of Electrical Engineering, Zhejiang University. From June 2009 to July 2010, he was a post doctor in the Electrical Engineering and Computer Science Department, the University of Central Florida, Orlando, FL, USA. His research interests include power system stability analysis and control, and renewable energy integration.

Xuhua FU is currently a senior engineer in Zhejiang Power Economic Research Institute of State Grid. His research interests include power system operation and planning, renewable energy integration. 